\title{
Ambra1 in autophagy and apoptosis: Implications for cell survival and chemotherapy resistance (Review)
}

\author{
WEI-LIANG SUN \\ Department of Internal Medicine-Oncology, The First Affiliated Hospital, \\ Guangxi Medical University, Nanning, Guangxi 530021, P.R. China
}

Received April 1, 2015; Accepted May 5, 2016

DOI: $10.3892 / \mathrm{ol} .2016 .4644$

\begin{abstract}
Increasing studies suggest that autophagy has a protective role in cancer treatment and may even be involved in chemotherapy resistance. Nevertheless, the mechanism of autophagy in cancer treatment and drug resistance has not yet been established. There is a complex association between autophagy and apoptosis. Accordingly, these two processes can mutually regulate and transform to determine the fate of a cell, depending on the context. Activating molecule in Beclin 1-regulated autophagy protein 1 (Ambra1) is an important factor at the crossroad between autophagy and apoptosis The expression level and intracellular distributions of Ambra1 may control the balance and conversion between autophagy and apoptosis, and modify the effectiveness of chemotherapy. Therefore, Ambral may provide a novel target for cancer treatment, particularly for overcoming anticancer drug resistance. The present review focuses on the role of Ambra1 in autophagy and apoptosis and assesses the implications for cell survival and chemotherapy resistance.
\end{abstract}

\section{Contents}

1. Introduction

2. Autophagy process and its dual role in cell death and survival

Correspondence to: Dr Wei-Liang Sun, Department of Internal Medicine-Oncology, The First Affiliated Hospital, Guangxi Medical University, 6 Shuangyong Road, Nanning, Guangxi 530021, P.R. China

E-mail: sw120022001@hotmail.com

Abbreviations: Ambra1, activating molecule in Beclin 1-regulated autophagy protein 1; ATG, autophagy-related gene; mTOR, mammalian target of rapamycin; ULK1, Unc-51 like kinase-1; ER, endoplasmic reticulum; DLC1/2, dynein light chain 1/2; Vps34, vacuolar protein-sorting protein 34 ; Mito-Bcl-2, Bcl-2 located at the mitochondria; ER-Bcl-2, Bcl-2 resided at the endoplasmic reticulum

Key words: activating molecule in beclin 1-regulated autophagy protein 1 , autophagy, apoptosis, chemotherapy, resistance
3. Autophagy in cancer therapeutic responsiveness and chemotherapy resistance

4. Ambra1 is a positive factor of autophagy

5. Ambral is a negative factor of apoptosis execution

6. Ambra1 in cell survival and implications for chemotherapy resistance

7. Conclusion

\section{Introduction}

Macroautophagy, which is also referred to as autophagy, is a protein degradation process in eukaryotic cells. The role of autophagy in cancer treatment has been extensively studied, yet the results remain controversial. Although certain studies indicate that autophagy participates in chemotherapy resistance, the mechanism is not yet clear (1). A recent series of studies suggest that activating molecule in Beclin 1-regulated autophagy protein 1 (Ambral) is an important factor in regards to the association between autophagy and apoptosis, and may control the reciprocal conversion between the two processes to decide the resulting cell death or survival (2-4). Therefore, Ambra1 may be an important factor of autophagy involved in cancer treatment and chemotherapy resistance. The present review focuses on the role of Ambral in autophagy and apoptosis and assesses the implications for cell survival and chemotherapy resistance.

\section{Autophagy process and its dual role in cell death and Survival}

Autophagy is an evolutionarily conserved lysosome-dependent cellular catabolic degradation process in eukaryotic cells (5). In general, basal autophagy exists in cells to maintain cellular homeostasis through the degradation of long-lived proteins, protein aggregates and damaged organelles. However, autophagy is rapidly upregulated under adverse conditions, including nutrient deprivation, hypoxia, radiation and anticancer drugs, to recycle energy and supply macromolecules for biosynthesis, leading to the cells adapting to the stress and survival (5-13). Three major types of autophagy have been reported, including macroautophagy, microautophagy and chaperone-mediated autophagy (CMA) (10). Autophagy is highly regulated by a series of autophagy-related genes (ATGs) 
that are essential for the formation, maturation and traffic of autophagosomes $(8,14)$. At present, $>30$ ATGs have been identified in yeast, the majority of which have a mammalian homolog (15).

Several complexes are essential at the initial stage of autophagy, including the mammalian target of rapamycin (mTOR) complex, Unc-51 like kinase-1 (ULK1) complex and the mammalian ortholog of the ATG6/vacuolar protein-sorting protein (Vps)30 (Beclin 1)-class III phosphatidylinositol 3-kinase (CIII PI3K/Vps34) complex (Vps34 complex) (16-18). The mTOR complex includes two distinct complexes, mTOR complex 1 (mTORC1) and mTOR complex 2 (mTORC2). The mTORC1 is a sensor of amino acids, adenosine triphosphate (ATP), growth factors and reactive oxygen species (ROS). Usually, mTORC1 combines with the ULK1 complex, leading to the phosphorylation of ULK1 and mammalian ATG13 (mATG13), thus deactivating the ULK1 complex and blocking autophagy (Fig. 1A) (16).

The ULK1 complex contains the mammalian ortholog of ATG1 (ULK1/2), mATG13, the mammalian ortholog of ATG17 (FIP200) and ATG101 (17). Under adverse conditions, the inactivation of mTORC1 results in the dephosphorylation of ULK1 and mATG13, prompting the activation of the ULK1 complex $(16,17)$. The activated ULK1 phosphorylates Beclin 1 and Ambral to promote the formation and activation of the Vps34 complex. In addition, the activated ULK1 directs the Vps34 complex to the endoplasmic reticulum (ER), where Vps34 catalyzes the transform of phosphatidylinositol (PI) into PI-3-phosphate, which recruits the specific autophagic proteins that are required for the formation of phagophores (Fig. 1B) (16-18).

The Vps34 complex is the core machinery of autophagy initiation, and is composed by Beclin 1, Vps34/CIII PI3K and Vps15 (19,20). Beclin 1, a B-cell lymphoma (Bcl)-2-homology (BH)3 domain only protein, is identified as a binding protein of $\mathrm{Bcl}-2$. It is an indispensible protein in mammalian autophagy induction (21-23). Beclin 1 contains three identified structural domains, including a BH3 domain, a central coiled-coil domain (CCD) and an evolutionarily conserved domain (ECD) $(18,24-26)$. On the one hand, Bcl-2 family members, including Bcl-2, Bcl-extra large and myeloid cell leukemia 1, interact with the $\mathrm{BH} 3$ domain to block the interaction of Beclin 1 with Vps34/CIII PI3K, decreasing Vps34/CIII PI3K activity and negatively regulating Beclin 1-dependent autophagy $(18,21-26)$ On the other hand, Beclin 1 directly binds to Vps34 with the ECD and CCD domains to form the Vps34 complex and arouse autophagic cascades (18). In addition, Beclin 1 regulates autophagy through various steps by associating with other specific proteins, including Ambra1, ATG14/Barkor, UV radiation resistance-associated gene and RUN and cysteine rich domain containing Beclin 1 interacting protein at the CCD domain $(18,22,26-28)$. In one previous study, Ambra1, a cofactor of Beclin 1, was also shown to be an inseparable part of the core Vps34 complex and a positive regulator of autophagy (2). Subsequently, the phagofores elongate and fuse to form double-membrane vesicles called autophagosomes. In the process of phagofore elongation, two ubiquitin-like conjugation systems are required, including the Atg12-Atg5 conjugation and ATG8/LC3-PE (phosphatidylethanolamine) system. Following the maturation of autophagosomes, the outer membrane eventually fuses with lysosomes to form autolysosomes $(8,14,15)$. The contents within the autolysosomes are digested by lysosomal hydrolases, the resultant macromolecules of which are recycled and catabolized, thus producing energy to aid the adaptation of cells to starvation or stress and contributing to cell survival $(6,12)$.

Although autophagy is primary to contribute to cell survival, in certain conditions, it can directly lead to cell death, particularly in the cells with apoptotic machinery deficiency $(6,12)$. This type of cell death is called autophagic cell death or type II programmed cell death $(6,12)$. At present, the mechanisms of cell death directly induced by autophagy have not been fully clarified. The recognized interpretation is that sustained autophagy leads to excessive degradation of necessary proteins and organelles, or induces the high threshold apoptosis (29). In cancer cells, autophagy has been previously indicated to be upstream to apoptosis in ER stress-induced death (30).

Therefore, autophagy is a highly regulated process that has a dual role in cell death or survival. In addition, the role of autophagy in determining the outcome of cells is dependent on the context and cell type.

\section{Autophagy in cancer therapeutic responsiveness and chemotherapy resistance}

Autophagy in cancer treatment. Previously, a growing body of evidence has revealed that a variety of cancer treatments, including chemotherapy, irradiation, endocrine therapy and molecular-targeted therapy, can induce autophagy in diverse cancer cell lines, and that induced-autophagy may be associated with therapeutic effects $(1,7,19)$. Unfortunately, the role of autophagy in cancer treatment remains controversial. Certain studies have shown that autophagy induced by anticancer treatment is a pro-death mechanism. For example, histone deacetylase inhibitor, suberoylanilide hydroxamic acid, 5-fluorouracil, sorafenib and imatinib, could respectively induce cell death by autophagy in the cells of breast cancer, colorectal cancer (CRC), hepatocellular carcinoma and glioma; while the inhibition of autophagy with chemical reagents, such as 3-methyladenine, or small interfering RNAs (siRNAs), including Beclin1 or ATG5, may suppress cell death (31-34). As a result, autophagy is beneficial to cancer treatment. On the contrary, increasing studies have shown that autophagy induced by anticancer treatment plays a protective role (35-55). Therefore, the blockage of autophagy is beneficial to cancer treatment. Based on the results, $>30$ clinical trials have been opened to investigate the effectiveness of chloroquine (CQ) or hydroxychloroquine (HCQ) plus or minus chemotherapeutic or targeted-drugs in human cancers (1). CQ and its derivative HCQ raise the lysosomal $\mathrm{pH}$ and ultimately inhibit the fusion between autophagosomes and lysosomes, thus preventing the maturation of autophagosomes into autolysosomes, and blocking a late step of autophagy $(56,57)$. Notably, numerous trials have supplied evidence of preliminary anticancer activity of CQ or HCQ (1).

Autophagy in chemotherapy resistance. Chemotherapy is one of the major means of cancer treatment. However, the resistance of cancer cells to drugs seriously limits their use. The mechanisms underlying drug resistance are numerous, 
A

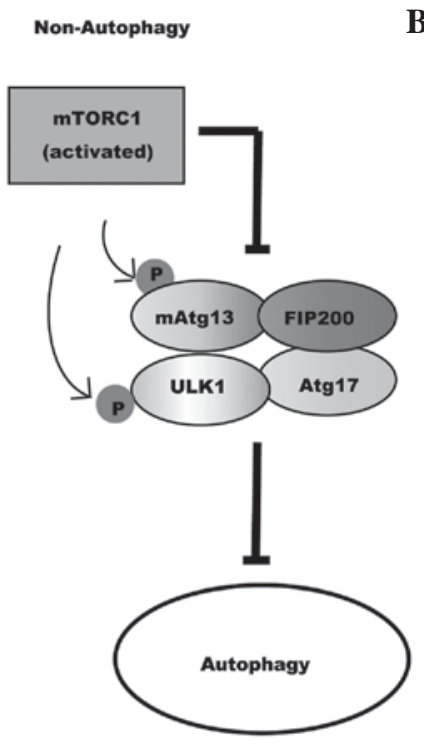

B

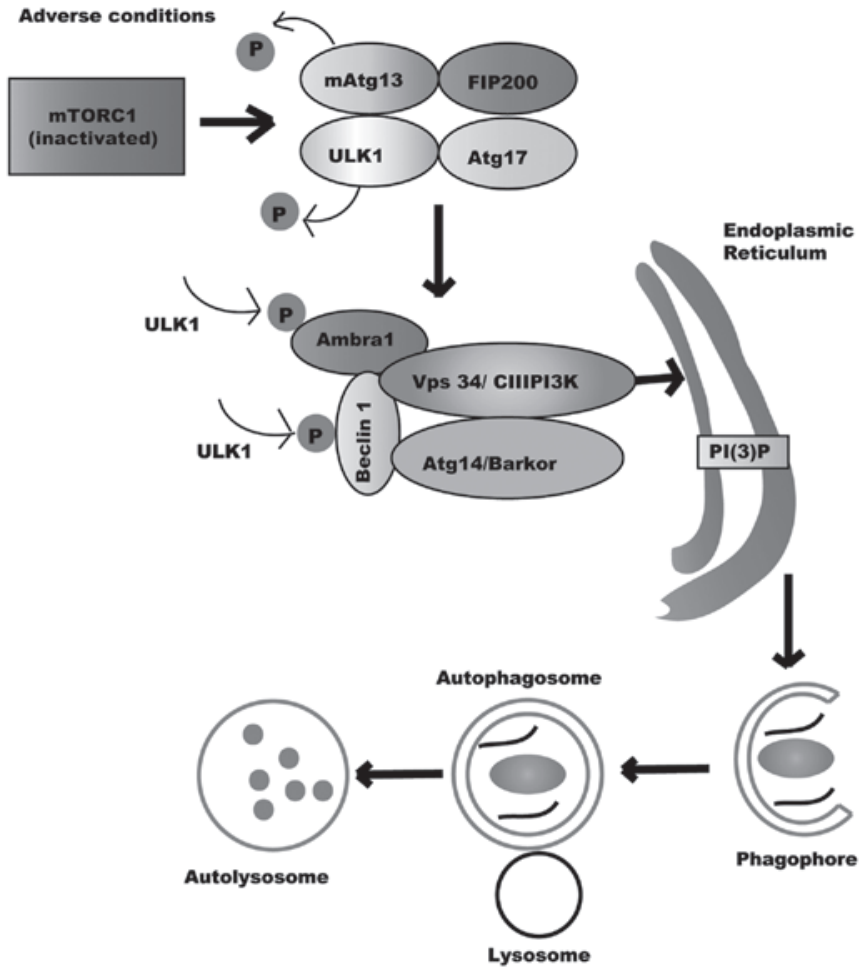

Figure 1. Autophagy process. (A) Non-autophagy, mTORC1 binds and deactivates ULK1 complex through phosphorylating ULK1 and mATG13, thus blocking autophagy. (B) Under adverse conditions, the inactivation of mTORC1 makes ULK1 and mATG13 dephosphorylate, prompting the activation of the ULK1 complex. Activated ULK1 then phosphorylates Beclin 1 and Ambra1, leading to the formation and activation of the Vps34 complex. Activated ULK1 directs the Vps34 complex to the endoplasmic reticulum, where Vps34 catalyzes the transformation of PI into PI-3-P, which recruits the specific autophagic proteins that are required for the formation of phagophores. Subsequently, the phagofores elongate and fuse to form autophagosomes. After the maturation of autophagosomes, the outer membrane of it eventually fuses with lysosomes to form autophagolysosomes. Lastly, the contents of the autolysosomes are digested by lysosomal hydrolases. mTORC1, mammalian target of rapamycin complex 1; ULK1, Unc-51 like kinase-1; mATG13, mammalian autophagy-related gene 13; Ambra1, activating molecule in Beclin 1-regulated autophagy protein 1; Vps34/CIII PI3K, class III phosphatidylinositol 3-kinase; ATG17, autophagy-related gene 17; FIP200, mammalian ortholog of ATG17; ATG14/Barkor, autophagy-related gene 14; PI(3)P, phosphatidylinositol-3-phosphate.

including reduced drug absorption, the reduced ability of drugs to eliminate cells due to diverse changes in cells, increased efflux pump and so on $(58,59)$. To date, these mechanisms are not entirely understood.

The protective role of autophagy in cancer treatment suggests that autophagy may be involved in anticancer drug resistance. In fact, the role of autophagy in anticancer drug resistance has been confirmed by the results from several breast cancer cell lines (60-65). Epirubicin can induce autophagy in MCF-7 cells, and the induced-autophagy protects the cells from death by blocking apoptosis (60). Similarly, this reagent also induces autophagy in derived MCF-7er cells (a type of induced epirubicin-resistant cell), and the inhibition of induced-autophagy by chemical inhibitors, such as baflomycin A1, or small hairpin RNAs (shRNAs), including sh-Beclin 1 and sh-ATG7, restores the sensitivity of MCF-7er cells to epirubicin through enhancing apoptosis (60). MCF-7er cells also generate resistance to paclitaxel (PTX) and vinorelbine (NVB), which suggests that the cells obtain a multidrug resistance (MDR) phenotype (61). Furthermore, the inhibition of PTX and NVB induced-autophagy makes the cells more sensitive to these drugs, indicating the autophagy is also involved in MDR development (61). In addition, Chittaranjan et al (62) indicated that the blockage of epirubicin-induced autophagy augments the anticancer effects of epirubicin in triple-negative breast cancer MDA-MB-231 cells, derived resistant MDA-MB-231-R8 cells and SUM159PTR75 cells. These results suggest that autophagy facilitates epirubicin resistance development by blocking apoptosis. Additionally, other studies also demonstrate that the induced-autophagy in various breast cancer cells contributes to the development of resistance to paclitaxel, tamoxifen or herceptin (63-65). Notably, these studies indicated that derived resistant breast cancer cells obtained an increased capability for autophagy compared with the parental cells, which further suggests that autophagy is involved in the drugs resistance. Nevertheless, the molecular mechanisms of autophagy in drug resistance are complex and not yet established. A number of factors or signaling pathways may participate in the process, including epidermal growth factor receptor signaling, the PI3K/protein kinase B/mTOR axis, tumor protein 53 and mitogen-activated protein kinase 14/p38 signaling (1). In the future, additional studies in vitro and in vivo are required to confirm the role of autophagy in drug resistance and to explore the underlying mechanisms.

\section{Ambra1 is a positive factor of autophagy}

Ambra1 is a Beclin 1-interacting protein that contains a WD40 domain. It is primarily expressed in neural tissues and is essential for normal neural tube development (3). In mouse embryos, the functional deficiency of Ambra1 results in serious neural 


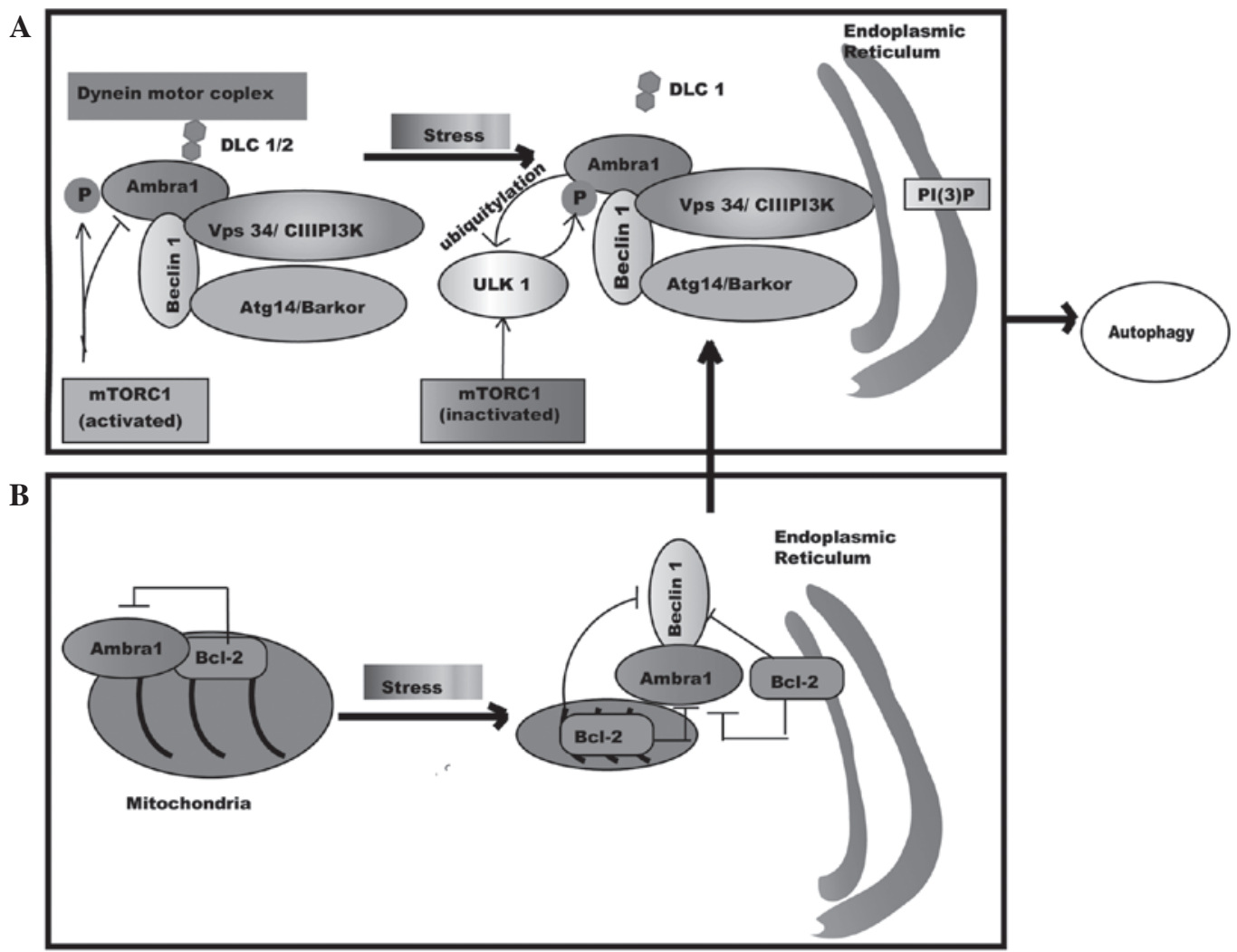

Figure 2. Ambra1 in autophagy. (A) Ambra1 physically interacts with mTORC1, deactivating mTORC1 by phosphorylation; however, the association between Ambra1 and the Beclin1-Vps34 complex is deactivated by binding to the dynein motor complex through interplay with DLC 1/2. While under stress settings, Ambra1 is activated through phosphorylation by ULK1, the association of Ambra1-DLC1 and the Beclin1-Vps34 complex is released from the dynein motor complex, and the core complex translocates to the ER and accelerates the formation of PI(3)P, which leads to autophagosome formation. (B) Ambra1 is docked at the mitochondria by Bcl-2. Under stress conditions, Ambral is separated from mito-Bcl-2 and relocated to the outer membrane of mitochondria. Ambra1 then competes with mito- and ER-Bcl-2 to bind Beclin1 and prompts Beclin 1-dependent autophagy. Ambra1, activating molecule in Beclin 1-regulated autophagy protein 1; mTORC1, mammalian target of rapamycin complex 1; ULK1, Unc-51 like kinase-1; Vps34/CIII PI3K, class III phosphatidylinositol 3-kinase; ATG14/Barkor, autophagy-related gene 14; DLC, dynein light chain; PI(3)P, phosphatidylinositol-3-phosphate; ER, endoplasmic reticulum; Bcl-2, B cell lymphoma-2.

tube defects, which are associated with autophagy impairment, accumulation of ubiquitinated proteins, unbalanced cell proliferation and excessive apoptotic cell death (3). The overexpression of Ambra1 in rapamycin-treated human fibroblast 2FTGH cells has been shown to significantly increase basal and rapamycin-induced autophagy (3). On the contrary, downregulation of Ambra1 results in an evident decrease in autophagy induced by rapamycin and nutrient deficiency (3). Therefore, Ambral is a positive factor of autophagy induction. The functions of Ambral in autophagy regulation are mainly through interaction with mTORC1, ULK1, Beclin 1, dynein light chain 1/2 (DLC 1/2) and Bcl-2 located at the mitochondria (mito-Bcl-2) (2,66-69).

Normally, Ambra1 physically interacts with mTORC1, which results in Ambral deactivation by phosphorylation at Ser 52 (Fig.2A) (67). However, under stress conditions, mTORC1 inactivates and ULK1 activates, which results in the activation of Ambral and Beclin 1 through phosphorylation and leads to autophagy induction (Fig. 1B) (67). Recently, Nazio et al (67) have found that Ambra1 is a ULK1-binding partner that is required for ULK1 stability and kinase activity. On autophagy induction, Ambra1 mediates ULK1 Lys-63-linked ubiquitylation through interaction with the E3-ligase tumor necrosis receptor associated factor 6 (Fig. 2A) (67). Ubiquitylation enhances ULK1 stability, kinase activity and self-association; therefore, there is a positive regulation loop between Ambra1 and ULK1 in autophagy regulation (67).

As a binding protein of Beclin 1, Ambral can directly interact with it at the CCD domain; equally, Beclin 1 can bind to Ambra1 at the central region. The two proteins are the primary elements of the Vps34 complex. In addition, Ambra1 can modify the function of Beclin 1 through regulation the ubiquitylation of it at lysine 437 (68). The Ambra1-damage specific DNA binding protein 1-Cullin-4A complex is an E3 ligase for K63-linked ubiquitylation of Beclin $1(70,71)$. The ubiquitination of Beclin 1 enhances the association of it with Vps34 and promotes the activation of Vps34, which is required for starvation-induced autophagy (70). Furthermore, studies have shown that the downregulation of Ambra1 leads to a reduced capability of Beclin 1 to interact with Vps34, and a decrease in Vps34 activation $(20,72)$. Therefore, Ambra1 triggers autophagy through interaction with Beclin 1 to activate Vps34 kinase and to promote Vps34 complex formation at the beginning stages of autophagy.

Ambral has been found to dynamically bind DLC 1/2, which leads the Ambra1-Beclin 1-Vps34 complex to tether to the dynein motor complex, resulting in core complex deactivation $(2,66,68)$. During autophagy, Ambra1 is phosphorylated by 


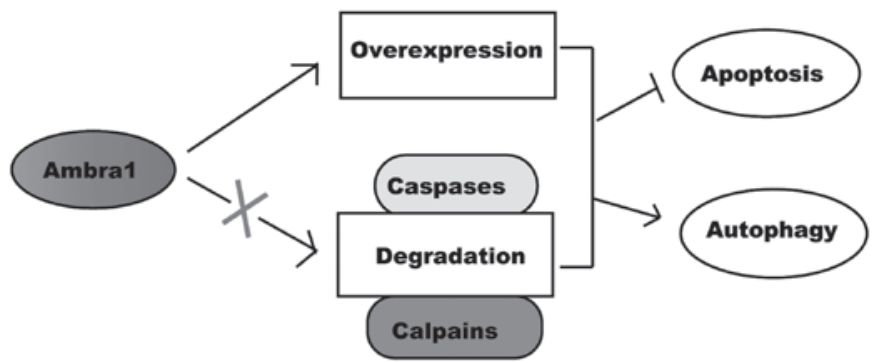

Figure 3. Ambra1 in apoptosis. In cells, the overexpression or damaged degradation of Ambra1 leads to its accumulation, making cells avoid apoptosis and more easily undergo autophagy and survival. Ambra1, activating molecule in Beclin 1-regulated autophagy protein 1.

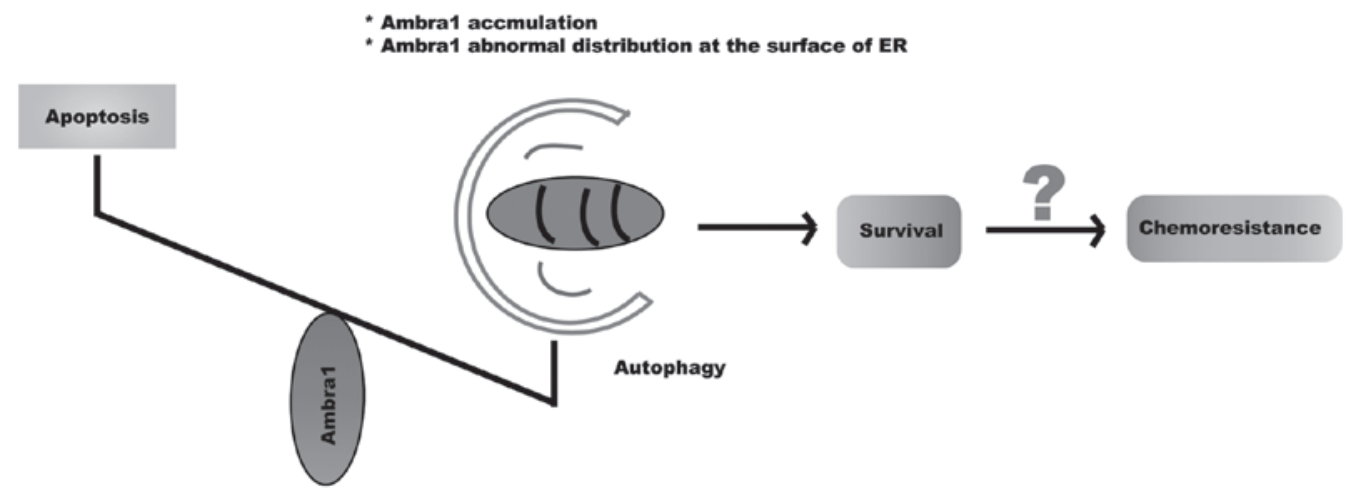

Figure 4. Ambra1 in chemotherapy resistance. In resistant cells, Ambra1 may accumulate due to overexpression, damaged degradation or abnormal distribution at the surface of the ER, which shifts the balance towards autophagy and accelerates the occurrence of chemoresistance. Ambra1, activating molecule in Beclin 1-regulated autophagy protein 1; ER, endoplasmic reticulum.

ULK1 and the Ambra1-DLC1 and Beclin 1-Vps34 complexes are released from the dynein motor complex. Subsequently, the core complex translocates to the ER and initiates autophagic cascades (Fig. 2A). In addition, Ambra1 can also regulate autophagy through dynamic combination with mito-Bcl-2 (68). Usually, a pool of Ambra1 proteins is docked at the mitochondria by Bcl-2; however, these proteins remain separate from $\mathrm{Bcl}-2$ and relocate on the outer membrane of the mitochondria when autophagy occurs (68). Ambral then competes with mito-Bcl2 and $\mathrm{Bcl}-2$ resided at the endoplasmic reticulum to bind Beclin 1, thus prompting Beclin 1-dependent autophagy (Fig. 2B). Previously, Ambra1-Bcl2 interaction has been found to decrease at the mitochondria, whereas Ambra1-Beclin 1 interaction increases at the mitochondria and ER following the initiation of autophagy (68). Notably, the Ambra1-Bcl-2 interaction at the mitochondria is disrupted by autophagy and apoptosis induction (68). As a result, the dynamic subcellular localization of Ambra1 is also an important factor of autophagy regulation.

In summary, Ambra1 is a pro-autophagy factor, and the function of Ambral in autophagy induction is a complex process that requires additional studies.

\section{Ambra1 is a negative factor of apoptosis execution}

Ambral has been found to be important for apoptosis execution (66). As previously mentioned, the functional deficiency of Ambral in mouse embryos leads to excessive apoptotic cell death (3). Similarly, the downregulation of Ambra1 in adult neural stem cells results in an increase in basal apoptosis and an augmented sensitivity to DNA-damage-induced death (73). In addition, in 2FTG cells and CRC SW620 cells, the downregulation of Ambra1 with siRNA results in increased sensitivity of the cells to staurosporine- and etoposide-induced apoptosis, while the overexpression of Ambra1 makes the cells undergo autophagy and survival more easily (4). Therefore, the expression of Ambra1 is negatively associated with apoptosis. Pagliarini et al (74) have found that Ambral is rapidly degraded by caspases and calpains when apoptosis is induced by staurosporine in human fibroblast 2 FTGH cells. The phenomenon has also been observed in SW620 cells during etoposide-induced apoptosis (4). Previously, caspases have been found to be responsible for Ambral cleavage at the D482 site, whereas calpains are involved in complete Ambral degradation (74). In addition, caspase-uncleavable Ambra $1^{\mathrm{D} 482 \mathrm{~A}}$ mutant $2 \mathrm{FTGH}$ cells confer increased resistance to staurosporine- and etoposide-induced cell death (74). Thus, Ambra1 is an important target of apoptotic proteases resulting in the dismantling of the autophagic machinery. Therefore, the overexpression or damaged degradation of Ambra1 leads to its accumulation, and makes the cells avoid apoptosis and more easily undergo autophagy and survival (Fig. 3). Furthermore, Ambral preferentially binds the pool of mito- $\mathrm{Bcl}-2$ proteins, and the Ambra1-Bcl-2 interaction is disrupted by the induction of apoptosis and autophagy, which indicates that Ambral has a double function in the regulation of autophagy and apoptosis (68). During autophagy induction, mito-Bcl-2 separates from Ambral leading to the increased release of $\mathrm{Bcl}-2$, which may enhance the anti-apoptotic function of Bcl-2 (69). 
Therefore, Ambra1 is an important factor of apoptosis execution, and the level at which it is expressed will determine whether the cell undergoes autophagy or apoptosis.

\section{Ambra1 in cell survival and implications for chemother- apy resistance}

Previously, studies have indicated that there is a complex association between autophagy and apoptosis $(13,75)$. For example, the important apoptotic proteins, including Bcl-2 family members and caspases, participate in the regulation of autophagy, whereas numerous autophagic proteins, including Beclin 1, Ambra1, ATG5 and ATG12, are involved in apoptosis execution (75). Therefore, these two processes can mutually regulate and transform, depending on the context. A study by Hou et al (76) demonstrated the autophagic degradation of active caspase- 8 during tumor necrosis factor superfamily member 10-induced autophagy, indicating that only one of these two processes can prevail at a time.

As has been previously shown, Ambral is an important factor at the crossroad between autophagy and apoptosis, which may control the balance and conversion between autophagy and apoptosis. Ambra1 may primarily play a pro-survival role due to the positive induction of autophagy, which has been previously demonstrated in in vivo (mouse embryos) and in vitro (2FTG cells, SW620 cells and neural stem cells) studies $(3,4,73,74)$. According to these results, the accumulation of Ambral in cells will promote autophagy occurrence and suppress apoptosis execution, which may decrease the effectiveness of chemotherapy. Therefore, Ambral may be a negative factor in cancer treatment and prognosis, and involved in drug resistance. Previously, it has been reported that Ambral is expressed in $\sim 63.9 \%$ patients with pancreatic ductal adenocarcinoma (77). Furthermore, the increased expression of Ambral is marginally associated with perineural invasion $(\mathrm{P}=0.063)$, which is a negative prognostic factor of cancer therapy, and significantly associated with poor overall survival $(\mathrm{P}=0.032)(77)$. Nevertheless, no more data currently exists on the expression of Ambral in other human cancers and its role in chemotherapy resistance. In addition, the intracellular distribution and interplay of Ambral with other proteins, such as Bcl-2, can also modify the function of Ambral and control the conversion between autophagy and apoptosis to determine cell death or survival (68). Therefore, Ambral is an important factor in deciding the fate of cells. Increased levels of Ambral due to overexpression or damaged degradation, in addition to abnormal distribution and interaction with other proteins, may shift the balance towards autophagy instead of apoptosis in order to help the cells to survive, thus leading to the resistance to anticancer drugs.

\section{Conclusion}

It is not surprising that autophagy is involved in the resistance of cancer to anticancer drugs, due to its dual role in cell death and survival. However, the current knowledge of the molecular mechanisms of autophagy in drug resistance remains superficial. Ambral is an important factor in regards to the association between autophagy and apoptosis, and for switching between these two processes. The accumulation of Ambral promotes autophagy occurrence, protecting cells from apoptosis. In addition, the abnormal distribution and interplay of Ambral with other proteins can also modify its function. Therefore, Ambral may be a novel target for cancer treatment, and involved in chemotherapy resistance. Based on these findings, it is possible that, in resistant cells, Ambra1 demonstrates accumulation or abnormal distribution at the surface of the ER, which tips the balance towards autophagy and accelerates cell survival, thus decreasing the effectiveness of chemotherapy and generating resistance to the drugs (Fig. 4). In the future, rigorous studies in vivo and in vitro are required to confirm this theory and to investigate the potential mechanism. Furthermore, additional studies may be helpful for understanding the mechanism of drugs resistance, and supply novel strategies for cancer treatment, particularly for overcoming chemotherapy resistance in a clinical setting.

\section{Acknowledgements}

The present study is supported by grants from the National Natural Science Foundation of China (Beijing, China; grant no. 81360340) and the Wu Jieping Medical Foundation clinical research special fund (Beijing, China; grant no. 320.6750.12689).

\section{References}

1. Sui X, Chen R, Wang Z, Huang Z, Kong N, Zhang M, Han W, Lou F, Yang J, Zhang Q, et al: Autophagy and chemotherapy resistance: A promising therapeutic target for cancer treatment. Cell Death Dis 4: e838, 2013.

2. Fimia GM, Corazzari M, Antonioli M and Piacentini M: Ambra1 at the crossroad between autophagy and cell death. Oncogene 32: 3311-3318, 2013.

3. Fimia GM, Stoykova A, Romagnoli A, Giunta L, Di Bartolomeo S, Nardacci R, Corazzari M, Fuoco C, Ucar A, Schwartz P, et al: Ambral regulates autophagy and development of the nervous system. Nature 447: 1121-1125, 2007.

4. Gu W, Wan D, Qian Q, Yi B, He Z, Gu Y, Wang L and He S: Ambral is an essential regulator of autophagy and apoptosis in SW620 cells: Pro-survival role of Ambra1. PLoS One 9: e90151, 2014.

5. Klionsky DJ: Autophagy: From phenomenology to molecular understanding in less than a decade. Nat Rev Mol Cell Biol 8: 931-937, 2007.

6. Kroemer $\mathrm{G}$ and Jäättelä M: Lysosomes and autophagy in cell death control. Nat Rev Cancer 5: 886-897, 2005.

7. Kondo Y, Kanzawa T, Sawaya R and Kondo S: The role of autophagy in cancer development and response to therapy. Nat Rev Cancer 5: 726-734, 2005.

8. Xie ZP and Klionsky DJ: Autophagosome formation: Core machinery and adaptation. Nat Cell Biol 9: 1102-1109, 2007.

9. Levine B: Cell biology: Autophagy and cancer. Nature 446: 745-747, 2007.

10. Ravikumar B, Futter M, Jahreiss L, Korolchuk VI, Lichtenberg M, Luo S, Massey DC, Menzies FM, Narayanan U, Renna M, et al: Mammalian macroautophagy at a glance. J Cell Sci 122: 1707-1711, 2009.

11. Baehrecke EH: Autophagy: Dual roles in life and death? Nat Rev Mol Cell Biol 6: 505-510, 2005.

12. Levine B and Yuan J: Autophagy in cell death: An innocent convict? J Clin Invest 115: 2679-2688, 2005.

13. Maiuri MC, Zalckvar E, Kimchi A and Kroemer G: Self-eating and self-killing: Crosstalk between autophagy and apoptosis. Nat Rev Mol Cell Biol 8: 741-752, 2007.

14. Mizushima N, Yoshimori T and Ohsumi Y: The role of Atg proteins in autophagosome formation. Annu Rev Cell Dev Biol 27: 107-132, 2011.

15. Rubinsztein DC, Shpilka T and Elazar Z: Mechanisms of autophagosome biogenesis. Curr Biol 22: R29-R34, 2012.

16. Dunlop EA and Tee AR: mTOR and autophagy: A dynamic relationship governed by nutrients and energy. Semin Cell Dev Biol 36: 121-129, 2014. 
17. Wong PM, Puente C, Ganley IG and Jiang XJ: The ULK1 complex: Sensing nutrient signals for autophagy activation. Autophagy 9: 124-137, 2013.

18. Kang R, Zeh HJ, Lotze MT and Tang D: The Beclin 1 network regulates autophagy and apoptosis. Cell Death Differ 18: 571-580, 2011.

19. Lorin S, Hamaïb A, Mehrpour M and Codogno P: Autophagy regulation and its role in cancer. Semin Cancer Biol 23: 361-379, 2013.

20. Funderburk SF, Wang QJ and Yue Z: The Beclin 1-VPS34 complex-at the crossroads of autophagy and beyond. Trends Cell Biol 20: 355-362, 2010.

21. Liang XH, Jackson S, Seaman M, Brown K, Kempkes B, Hibshoosh $\mathrm{H}$ and Levine B: Induction of autophagy and inhibition of tumorigenesis by beclin 1. Nature 402: 672-676, 1999.

22. Maiuri MC, Criollo A, Tasdemir E, Vicencio JM, Tajeddine N, Hickman JA, Geneste O and Kroemer G: BH3-only proteins and $\mathrm{BH} 3$ mimetics induce autophagy by competitively disrupting the interaction between Beclin 1 and $\mathrm{Bcl}-2 / \mathrm{Bcl}-\mathrm{X}$ (L). Autophagy 3: 374-376, 2007.

23. Sinha S and Levine B: The autophagy effector Beclin 1: A novel BH3-only protein. Oncogene 27 (Suppl 1): S137-S148, 2008.

24. Fu LL, Cheng Y and Liu B: Beclin-1: Autophagic regulator and therapeutic target in cancer. Int J Biochem Cell Biol 45: 921-924, 2013.

25. Toton E, Lisiak N, Sawicka P and Rybczynska M: Beclin-1 and its role as a target for anticancer therapy. J Physiol Pharmacol 65 459-467, 2014.

26. He $\mathrm{C}$ and Levine B: The Beclin 1 interactome. Curr Opin Cell Biol 22: 140-149, 2010.

27. Matsunaga K, Saitoh T, Tabata K, Omori H, Satoh T, Kurotori N, Maejima I, Shirahama-Noda K, Ichimura T, Isobe T, et al: Two Beclin 1-binding proteins, Atg14L and Rubicon, reciprocally regulate autophagy at different stages. Nat Cell Biol 11: 385-396, 2009.

28. Itakura E, Kishi C, Inoue $\mathrm{K}$ and Mizushima N: Beclin 1 forms two distinct phosphatidylinositol 3-kinase complexes with mammalian Atg14 and UVRAG. Mol Biol Cell 19: 5360-5372, 2008.

29. Choi KS: Autophagy and cancer. Exp Mol Med 44: 109-120, 2012.

30. Corazzari M, Fimia GM and Piacentini M: Dismantling the autophagic arsenal when it is time to die: Concerted AMBRA1 degradation by caspases and calpains. Autophagy 8: 1255-1257, 2012.

31. Shingu T, Fujiwara K, Bögler O, Akiyama Y, Moritake K, Shinojima N, Yokoyama T and Kondo S: Inhibition of autophagy at a late stage enhances imatinib-induced cytotoxicity in human malignant glioma cells. Int J Cancer 124: 1060-1071, 2009.

32. Xiong HY, Guo XL, Bu XX, Zhang SS, Ma NN, Song JR, Hu F, Tao SF, Sun K, Li R, et al: Autophagic cell death induced by 5 -FU in Bax or PUMA deficient human colon cancer cell. Cancer Lett 288: 68-74, 2010.

33. Lee YJ, Won AJ, Lee J, Jung JH, Yoon S, Lee BM and Kim HS Molecular mechanism of SAHA on regulation of autophagic cell death in tamoxifen-resistant MCF-7 breast cancer cells Int J Med Sci 9: 881-893, 2012

34. Tai WT, Shiau CW, Chen HL, Liu CY, Lin CS, Cheng AL, Chen PJ and Chen KF: Mcl-1-dependent activation of Beclin 1 mediates autophagic cell death induced by sorafenib and SC-59 in hepatocellular carcinoma cells. Cell Death Dis 4: e485, 2013.

35. Li J, Hou N, Faried A, Tsutsumi S and Kuwano H: Inhibition of autophagy augments 5-fluorouracil chemotherapy in human colon cancer in vitro and in vivo model. Eur J Cancer 46: $1900-1909,2010$

36. de la Cruz-Morcillo MA, Valero ML, Callejas-Valera JL, Arias-González L, Melgar-Rojas P, Galán-Moya EM García-Gil E, García-Cano J and Sánchez-Prieto R: P38MAPK is a major determinant of the balance between apoptosis and autophagy triggered by 5 -fluorouracil: Implication in resistance. Oncogene 31: 1073-1085, 2012

37. Sasaki K, Tsuno NH, Sunami E, Tsurita G, Kawai K, Okaji Y, Nishikawa T, Shuno Y, Hongo K, Hiyoshi M, et al: Chloroquine potentiates the anti-cancer effect of 5-fluorouracil on colon cancer cells. BMC Cancer 10: 370, 2010.

38. Sasaki K, Tsuno NH, Sunami E, Kawai K, Hongo K, Hiyoshi M, Kaneko M, Murono K, Tada N, Nirei T, et al: Resistance of colon cancer to 5 -fluorouracil may be overcome by combination with chloroquine, an in vivo study. Anticancer Drugs 23: 675-682, 2012

39. Yang PM, Liu YL, Lin YC, Shun CT, Wu MS and Chen CC: Inhibition of autophagy enhances anticancer effects of atorvastatin in digestive malignancies. Cancer Res 70: 7699-7709, 2010.
40. Paillas S, Causse A, Marzi L, de Medina P, Poirot M, Denis V, Vezzio-Vie N,Espert L, ArzoukH,Coquelle A, et al: MAPK14/p38a confers irinotecan resistance to TP53-defective cells by inducing survival autophagy. Autophagy 8: 1098-1112, 2012.

41. Liu D, Yang Y, Liu Q and Wang J: Inhibition of autophagy by 3-MA potentiates cisplatin-induced apoptosis in esophageal squamous cell carcinoma cells. Med Oncol 28: 105-111, 2011

42. Ding ZB, Hui B, Shi YH, Zhou J, Peng YF, Gu CY, Yang H, Shi GM, Ke AW, Wang XY, et al: Autophagy activation in hepatocellular carcinoma contributes to the tolerance of oxaliplatin via reactive oxygen species modulation. Clin Cancer Res 17: 6229-6238, 2011 .

43. Guo XL, Li D, Sun K, Wang J, Liu Y, Song JR, Zhao QD, Zhang SS, Deng WJ, Zhao X, et al: Inhibition of autophagy enhances anticancer effects of bevacizumab in hepatocarcinoma. J Mol Med (Berl) 91: 473-483, 2013.

44. Shi YH, Ding ZB, Zhou J, Hui B, Shi GM, Ke AW, Wang XY, Dai Z, Peng YF, Gu CY, et al: Targeting autophagy enhances sorafenib lethality for hepatocellular carcinoma via ER stress-related apoptosis. Autophagy 7: 1159-1172, 2011.

45. Zhao M, Yang M, Yang L, Yu Y, Xie M, Zhu S, Kang R, Tang D, Jiang Z, Yuan W, et al: HMGB1 regulates autophagy through increasing transcriptional activities of JNK and ERK in human myeloid leukemia cells. BMB Rep 44: 601-606, 2011.

46. Han W, Pan H, Chen Y, Sun J, Wang Y, Li J, Ge W, Feng L, Lin X, Wang X, et al: EGFR tyrosine kinase inhibitors activate autophagy as a cytoprotective response in human lung cancer cells. PLoS One 6: e18691, 2011.

47. Wang Y, Peng RQ, Li DD, Ding Y, Wu XQ, Zeng YX, Zhu XF and Zhang XS: Chloroquine enhances the cytotoxicity of topotecan by inhibiting autophagy in lung cancer cells. Chin J Cancer 30: 690-700, 2011.

48. Kang R, Tang D, Schapiro NE, Livesey KM, Farkas A, Loughran P, Bierhaus A, Lotze MT and Zeh HJ: The receptor for advanced glycation end products (RAGE) sustains autophagy and limits apoptosis, promoting pancreatic tumor cell survival. Cell Death Differ 17: 666-676, 2010.

49. Shin SW, Kim SY and Park JW: Autophagy inhibition enhances ursolic acid-induced apoptosis in PC 3 cells. Biochim Biophys Acta 1823: 451-457, 2012.

50. Schoenlein PV, Periyasamy-Thandavan S, Samaddar JS, Jackson WH and Barrett JT: Autophagy facilitates the progression of ERalpha-positive breast cancer cells to antiestrogen resistance. Autophagy 5: 400-403, 2009.

51. O'Donovan TR, O'Sullivan GC and McKenna SL: Induction of autophagy by drug-resistant esophageal cancer cells promotes their survival and recovery following treatment with chemotherapeutics. Autophagy 7: 509-524, 2011.

52. Chen YS, Song HX, Lu Y, Li X, Chen T, Zhang Y, Xue JX, Liu H, Kan B, Yang G and Fu T: Autophagy inhibition contributes to radiation sensitization of esophageal squamous carcinoma cells. Dis Esophagus 24: 437-443, 2011.

53. Xu CX, Zhao L, Yue P, Fang G, Tao H, Owonikoko TK, Ramalingam SS, Khuri FR and Sun SY: Augmentation of NVP-BEZ235's anticancer activity against human lung cancer cells by blockage of autophagy. Cancer Biol Ther 12: 549-555, 2011.

54. Mirzoeva OK, Hann B, Hom YK, Debnath J, Aftab D, Shokat K and Korn WM: Autophagy suppression promotes apoptotic cell death in response to inhibition of the PI3K-mTOR pathway in pancreatic adenocarcinoma. J Mol Med (Berl) 89: 877-889, 2011.

55. Xu WH, Liu ZB, Hou YF, Hong Q, Hu DL and Shao ZM: Inhibition of autophagy enhances the cytotoxic effect of PA-MSHA in breast cancer. BMC Cancer 14: 273, 2014.

56. Fedorko M: Effect of chloroquine on morphology of cytoplasmic granules in maturing human leukocytes-an ultrastructural study. J Clin Invest 46: 1932-1942, 1967.

57. Klionsky DJ, Baehrecke EH, Brume JH, Chu CT, Codogno P, Cuervo AM, Debnath J, Deretic V, Elazar Z, Eskelinen EL, et al: A comprehensive glossary of autophagy-related molecules and processes (II edition). Autophagy 7: 1273-1294, 2011.

58. Gottesman MM: Mechanisms of cancer drug resistance. Annu Rev Med 53: 615-627, 2002.

59. Szakács G, Paterson JK, Ludwig JA, Booth-Genthe C and Gottesman MM: Targeting multidrug resistance in cancer. Nat Rev Drug Discov 5: 219-234, 2006.

60. Sun WL, Chen J, Wang YP and Zheng H: Autophagy protects breast cancer cells from epirubicin-induced apoptosis and facilitates epirubicin-resistance development. Autophagy 7: 1035-1044, 2011 
61. Sun WL, Lan D, Gan TQ and Cai ZW: Autophagy facilitates multidrug resistance development through inhibition of apoptosis in breast cancer cells. Neoplasma 62: 199-208, 2015.

62. Chittaranjan S, Bortnik S, Dragowska WH, Xu J, Abeysundara N, Leung A, Go NE, DeVorkin L, Weppler SA, Gelmon K, et al: Autophagy inhibition augments the anticancer effects of epirubicin treatment in anthracycline-sensitive and -resistant triple-negative breast cancer. Clin Cancer Res 20: 3159-3173, 2014.

63. Samaddar JS, Gaddy VT, Duplantier J, Thandavan SP, Shah M, Smith MJ, Browning D, Rawson J, Smith SB, Barrett JT and Schoenlein PV: A role for macroautophagy in protection against 4-hydroxytamoxifen-induced cell death and the development of antiestrogen resistance. Mol Cancer Ther 7: 2977-2987, 2008.

64. Vazquez-Martin A, Oliveras-Ferraros C and Menendez JA Autophagy facilitates the development of breast cancer resistance to the anti-HER2 monoclonal antibody trastuzumab. PLoS ONE 4: e6251, 2009.

65. Ajabnoor GM, Crook T and Coley HM: Paclitaxel resistance is associated with switch from apoptotic to autophagic cell death in MCF-7 breast cancer cells. Cell Death Dis 3: e260, 2012.

66. Di Bartolomeo S, Corazzari M, Nazio F, Oliverio S, Lisi G, Antonioli M, Pagliarini V, Matteoni S, Fuoco C, Giunta L, et al: The dynamic interaction of AMBRA1 with the dynein motor complex regulates mammalian autophagy. J Cell Biol 191: $155-168,2010$

67. Nazio F, Strappazzon F, Antonioli M, Bielli P, Cianfanelli V, Bordi M, Gretzmeier C, Dengjel J, Piacentini M, Fimia GM and Cecconi F: mTOR inhibits autophagy by controlling ULK1 ubiquitylation, self-association and function through AMBRA1 and TRAF6. Nat Cell Bio 15: 406-416, 2013.

68. Strappazzon F, Vietri-Rudan M, Campello S, Nazio F, Florenzano F, Fimia GM, Piacentini M, Levine B and Cecconi F: Mitochondrial BCL-2 inhibits AMBRA1-induced autophagy. EMBO J 30: 1195-1208, 2011.
69. Tooze1 SA and Codogno P: Compartmentalized regulation of autophagy regulators: Fine-tuning AMBRA1 by Bcl-2. EMBO J 30: 1185-1186, 2011.

70. Xia P, Wang S, Du Y, Zhao Z, Shi L, Sun L, Huang G, Ye B, Li C, Dai Z, et al: WASH inhibits autophagy through suppression of Beclin 1 ubiquitination. EMBO J 32: 2685-2696, 2013.

71. Xia P, Wang S, Huang G, Du Y, Zhu P, Li M and Fan Z: RNF2 is recruited by WASH to ubiquitinate AMBRA1 leading to downregulation of autophagy. Cell Res 24: 943-958, 2014.

72. Abrahamsen H, Stenmark H and Platta HW: Ubiquitination and phosphorylation of Beclin 1 and its binding partners: Tuning class III phosphatidylinositol 3-kinase activity and tumor suppression. FEBS Lett 586: 1584-1591, 2012.

73. Yazdankhah M, Farioli-Vecchioli S, Tonchev AB, Stoykova A and Cecconi F: The autophagy regulators Ambra1 and Beclin 1 are required for adult neurogenesis in the brain subventricular zone. Cell Death Dis 5: e1403, 2014.

74. Pagliarini V, Wirawan E, Romagnoli A, Ciccosanti F, Lisi G, Lippens S, Cecconi F, Fimia GM, Vandenabeele P, Corazzari $M$ and Piacentini $M$ : Proteolysis of Ambra1 during apoptosis has a role in the inhibition of the autophagic pro-survival response. Cell Death Differ 19: 1495-1504, 2012.

75. Booth LA, Tavallai S, Hamed HA, Cruickshanks $\mathrm{N}$ and Dent P: The role of cell signalling in the crosstalk between autophagy and apoptosis. Cell Signal 26: 549-555, 2014.

76. Hou W, Han J, Lu C, Goldstein LA and Rabinowich H: Autophagic degradation of active caspase-8: A crosstalk mechanism between autophagy and apoptosis. Autophagy 6: 891-900, 2010

77. Ko YH, Cho YS, Won HS, Jeon EK, An HJ, Hong SU, Park JH and Lee MA: Prognostic significance of autophagy-related protein expression in resected pancreatic ductal adenocarcinoma. Pancreas 42: 829-835, 2013. 